\title{
CrystEngComm
}

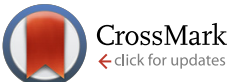

Cite this: CrystEngComm, 2016, 18, 2455

Received 3rd February 2016, Accepted 3rd March 2016

DOI: $10.1039 / \mathrm{c} 6 \mathrm{ce} 00289 \mathrm{~g}$

www.rsc.org/crystengcomm

\section{Crystal growth in gelled solution: applications to coordination polymers $\uparrow$}

\begin{abstract}
Silvia Rizzato, ${ }^{\star a}$ Massimo Moret, ${ }^{\star c}$ Marco Merlini, ${ }^{b}$ Alberto Albinati ${ }^{a}$ and Fabio Beghi ${ }^{a}$
Some critical issues concerning the complex synthesis and crystallization of designed coordination polymers are highlighted by using gels as diffusion media. The systematic use of gel methods in a variety of different crystallization configurations has been proven useful in revealing the field of existence and the stability hierarchy of competitive and concomitant crystalline phases, and also in discovering some important effects due to gel matrices. The most interesting phenomena observed are the modification and alteration of the macro- and microscopic morphology of crystals due to gel incorporation, which are responsible for changes in many properties and functionalities of these materials, opening up new possibilities for their applications in a variety of technological fields. In addition, a kinetic study of a gel grown polymeric system has also been conducted, demonstrating the continuous evolution of crystal morphology and its connection with the crystal growth process and growth mechanism.
\end{abstract}

\section{Introduction}

It is well known that the incorporation of organic materials in biogenic crystals results in a large variety of macro- and micro-morphologies, in distortions of crystal lattices, and in the formation of porous structures with pore sizes ranging from the micro- to the nanometer scale. ${ }^{1-3}$

In a similar way, synthetic calcite crystals grown in agarose hydrogel can show a porous internal structure whose origin has been hypothesized to be related to the incorporation of the gel fibres inside the crystals. ${ }^{4}$ However, until now, only a few synthetic crystals of different chemical nature (like small organic molecules or macromolecules) are known to trap gels, raising the question of how general this phenomenon might be.

For many crystals showing gel inclusion, specific investigations have demonstrated the uniform distribution of an incorporated gel matrix in the form of a network of nanofibres $^{5,6}$ and the real nature of crystals which are composed of equally oriented nanocrystallites. ${ }^{4,7,8}$ Moreover, although gels can interact chemically with growing crystals, affecting nucleation density or producing morphological effects, some

\footnotetext{
${ }^{a}$ Dipartimento di Chimica, Università degli Studi di Milano, Via Golgi 19, Milano, Italy.E-mail: silvia.rizzato@unimi.it

${ }^{b}$ Dipartimento di Scienze della Terra "Ardito Desio", Università degli Studi di Milano, Via Botticelli 23, Milano, Italy

${ }^{c}$ Dipartimento di Scienza dei Materiali, Università degli Studi di Milano Bicocca,

Via Roberto Cozzi 55, Milano, Italy

$\dagger$ Electronic supplementary information (ESI) available: Fig. S1-S5 and Table S1. See DOI: 10.1039/c6ce00289g
}

experimental evidences seem to support a physical mechanism for gel framework inclusion. ${ }^{9}$

Regardless of the entrapment mechanism and of the type and concentration of the included gel, crystals conserve their long range order that is their single crystal-nature. ${ }^{4,10}$ Correspondingly, the diffraction quality of crystals is substantially maintained, if not even improved, as in the case of some protein crystals. This is also because the trapping of gel into macromolecule crystal lattices can increase the mechanical stability of these materials and their resistance to dehydration, thus making their manipulation and characterization easier. ${ }^{11,12}$

Although the number of substances known to be able to incorporate gels is really limited and the underlying mechanism of the gel-incorporation phenomenon is not entirely clear, the gel technique seems to be a promising method to prepare single crystal composite systems and crystalline materials having a porous structure with a large internal surface area. ${ }^{13}$

Two further aspects to be considered are the possible effects of gels on macroscopic crystal shapes and also on the micromorphology of crystal surfaces,${ }^{14,15}$ and the capacity of gel media to promote the concomitant crystallization of different polymorphs with a monotropic relationship. ${ }^{14,16,17}$

Because of the similarities between both biological and inorganic systems with respect to their structural characteristics and functional properties, we have decided to apply the crystallization in gel technique to study a special class of compounds known as "coordination polymers". These can be considered as crystalline hybrid organic-inorganic materials showing an array of interesting physical and chemical 
properties with potential applications in many areas, such as gas storage and separation, catalysis, molecular sensing, ionexchange and drug delivery. ${ }^{18,19}$

Coordination polymers are usually built by self-assembly of metal-based nodes and organic bridging connectors to give networks with different dimensionalities and topologies that are usually structurally characterized by single crystal X-ray diffraction.

Actually, full and efficient control over the total design of a network structure cannot be achieved yet. An effective synthetic strategy based on a rational choice of the nature of metal centres and donor atoms, the length and geometry of bridging groups, the size and shape of counterions, etc. has been shown to work well. ${ }^{20,21}$ Nevertheless, significant challenges remain in order to obtain a complete understanding of the subtle driving factors operative during the self-assembly of these polymeric materials. As a consequence, in many cases, several polymeric species with different L/M (ligand to metal) stoichiometries, dimensionalities, topologies and porosities can be obtained under slightly different reaction conditions starting from the same ligand-metal salt pair. ${ }^{22}$

To gain further insight into the overall process, including better control of the final composition of competitive multiphase systems, a deep investigation into the crystal formation and growth should be carried out, following a more systematic investigation method.

In a previous work, we already adopted a more rational approach by studying the influence of a metal/ligand molar ratio and of absolute concentration on the composition and the structure of equilibrium products in order to obtain complete control of the outcome of network synthesis and also to induce specific solvent-mediated phase transitions. ${ }^{23}$

In that case, thanks to the knowledge acquired on the system's behaviour, we were able to produce a whole solubility phase diagram in a single crystallization trial by using the double diffusion gel technique.

Based on our previous observations, we decided to explore the possibility of using a variety of gels and resin-like systems as a dispersion matrix in crystallization experiments of coordination polymers, not only to increase the size and quality of crystals, but also as a useful medium useful to check for the existence of new phases, to improve the stability of porous crystals and to modify the macroscopic morphology and the microscopic structure of crystals.

\section{Experimental section}

\subsection{Materials and methods}

All the reagents (metal salts: $\mathrm{CdCl}_{2} \cdot 2.5 \mathrm{H}_{2} \mathrm{O}, \mathrm{Cu}\left(\mathrm{SO}_{3} \mathrm{CF}_{3}\right)_{2}$; ligands: bpp = 1,3-bis(4-pyridyl)propane, bpy = 4,4'-bipyridine; gels: agarose (EEO 0.09-0.13, gel point $36{ }^{\circ} \mathrm{C}$, gel strength $\geq 1200 \mathrm{~g} \mathrm{~cm}^{-2}$ at $1 \%$ gel (Sigma-Aldrich)); tetramethoxysilane (TMS) (purum $\geq 98.0 \%$ Sigma-Aldrich)) and solvents employed were commercially available, high-purity materials, and were used as supplied without further purification apart from the bpp ligand which was purified by sublimation.
Crystallization of $\left[\mathrm{Cu}(\mathrm{bpy})_{2}\left(\mathrm{SO}_{3} \mathrm{CF}_{3}\right)_{2}\right] \cdot 2 \mathrm{H}_{2} \mathrm{O} \cdot \mathrm{THF}$ (1). Crystals of a 3D-polymeric Cu-bpy system were grown by using the gel double diffusion technique. ${ }^{24}$ Crystallization experiments were carried out by using $\mathrm{U}$-shaped tubes $(4 \mathrm{~mm}$ i.d. and $100 \mathrm{~mm}$ long with two identical arms of $30 \mathrm{~mm}$ in length) ${ }^{25}$ filled up with low density silica gel prepared by using TMS as the gelling agent. A mixture of a liquid TMS reagent with water and THF solution $(15 \%, 15 \%$ and $70 \%$ in volume, respectively) was stirred until it became homogeneous (about 1 hour at room temperature). The solution was poured in the U-tubes and the gel was allowed to set. After one week, solutions of the reagents $(\sim 0.5 \mathrm{~mL})$ of appropriate concentrations were layered above the gel on opposite arms of the tubes. Equimolar $(0.200$ and $0.100 \mathrm{M})$ solutions of bpy in THF and copper salt $\left(\mathrm{Cu}\left(\mathrm{SO}_{3} \mathrm{CF}_{3}\right)_{2}\right)$ in water were employed. In order to demonstrate the reproducibility of the results, the experiments were repeated three times for each concentration.

Crystallization screening in gel for the $\mathbf{C d C l}_{2}$-bpp system. Short crystallization screening in gel for a binary $\mathrm{CdCl}_{2}$-bpp system was accomplished using the single gel diffusion technique. All the crystallization trials were performed in a glass tube with a length of $100 \mathrm{~mm}$ and diameter of $10 \mathrm{~mm}$ by layering a methanolic solution of the metal salt $\left(\mathrm{CdCl}_{2}\right.$ $\cdot 2.5 \mathrm{H}_{2} \mathrm{O}$ ) over a gelified solution of the ligand in water. Agarose powder was used as the gelifying medium. The test tubes were half-filled with the gel and the other half with the solution. The screening process was performed by changing the concentrations of the reactants $([\mathrm{L}]=30 \mathrm{mM}, 20 \mathrm{mM}, 10$ $\mathrm{mM})$, the gel density $\left(1 \%, 0,5 \%, 0,1 \% \mathrm{~m}(\mathrm{~g}) \mathrm{V}(\mathrm{mL})^{-1}\right.$ and the metal-ligand molar ratio $([\mathrm{M}] /[\mathrm{L}]: 1: 1,1: 2,1: 3)$. All the samples were left to stand at room temperature and were constantly monitored by using an optical microscope coupled to a CCD camera until equilibrium conditions were reached. On the basis of the obtained results, a phase diagram was built as a function of metal salts and ligand concentrations.

Preparation of powder samples for the $\mathbf{C d C l}_{2}$-bpp system. Microcrystalline powder samples were prepared from metha$\mathrm{nol} /$ water solution $(50: 50 \mathrm{v} / \mathrm{v})$ by mixing the metal salt $\left(\mathrm{CdCl}_{2}\right.$ $\cdot 2.5 \mathrm{H}_{2} \mathrm{O}$ ) and the ligand (bpp) at different molar ratios $(1: 1$, $1: 2,1: 3)$ and at several initial total concentrations ranging from 20 to $80 \mathrm{mM}$. The solutions were stirred at room temperature for at least two hours. The precipitate was then recovered by filtration through a Buchner funnel and dried in air.

X-ray powder diffraction spectra of the samples were collected on a Philips PW 3050 vertical scan diffractometer. The nature of the bulk precipitates was investigated by comparing the experimental patterns with those simulated from the single crystal data of 3 and 4 .

\subsection{High-pressure data}

In situ high-pressure single-crystal X-ray diffraction experiments were performed at the ID09A beamline of the European Synchrotron Radiation Facility (ESRF) in Grenoble, 
France. Dark blue euhedral crystals, approximately $80 \times 80 \times$ $50 \mu^{3}$ in size, were selected for the high-pressure experiments. A single crystal of 1 was loaded, along with a few ruby chips as $P$-calibrants ( $P$-precision between 0.02 and 0.09 $\mathrm{GPa}),{ }^{26,27}$ in a membrane-driven diamond anvil cell (DAC), mounting Boehler-Almax-design diamonds (culet diameter: $600 \mu \mathrm{m})$. A P-chamber $(250 \mu \mathrm{m}$ in diameter) was drilled by spark-erosion in steel gaskets, previously pre-indented to $c a$. $130 \mu \mathrm{m}$. A series of in situ $\mathrm{H}$ P-experiments were performed using silicone oil as the $P$-transmitting, "non-penetrating" fluid.

The experimental setup was based on that reported by Merlini and Hanfland: ${ }^{28}$ a parallel monochromatic beam with $E=30 \mathrm{keV}(\lambda=0.4140 \AA)$ was vertically focused using a spherical mirror and horizontally using a bent $\mathrm{Si}-(111)$ monochromator. The beam size on the sample was apx. $30 \times 30$ $\mu \mathrm{m} .{ }^{2}$ Diffraction patterns were collected using a MAR555 flatpanel detector. At any pressure point, the same data collection protocol was applied: $\Omega$-rotation of the DAC between $-30^{\circ}$ and $+30^{\circ}$ with $1^{\circ}$ of rotation step and $1 \mathrm{~s}$ per frame of exposure time. The collected diffraction patterns were analyzed using the Crysalis software. ${ }^{29}$ The unit-cell parameters were determined, from any dataset, by least-squares refinement based on the position of the diffracted peaks.

\subsection{Kinetic growth studies}

The experiment was performed inside a home-made optical cell consisting of two $2.6 \times 7.6 \mathrm{~cm}^{2}$ glass plates separated by a U-shaped rubber film which is $0.15 \mathrm{~cm}$ thick and $0.6 \mathrm{~cm}$ wide. The cell was half-filled with a warm ligand aqueous solution $([\mathrm{L}]=20.0 \mathrm{mM})$ containing agarose. Once the gel was cooled and set, an aqueous solution of $\mathrm{CdCl}_{2} \cdot 2.5 \mathrm{H}_{2} \mathrm{O}$ was layered on it until the top of the cell was reached. After closing its upper edge with adhesive tape, the glass cell was placed under a stereoscopic microscope (Olympus SZX16) equipped with a CCD camera (Lumera Infinity 1-3C) and a time-lapse video recorder. The images were acquired at a decreasing temporal sampling rate over time and analysed using the Infinity Analyze software (version 6.0.0). The growth rates have been obtained by measuring the variation of the linear size of the crystal on the video and converting it to a real value by using a previously determined calibration factor.

\subsection{Diffraction studies}

The elongated and partially deformed bipyramidal crystals of compound 3 grown at the sol-gel interface were analysed by $\mathrm{X}$-ray diffraction in order to confirm the single crystal nature of the samples and to compare the quality of the two morphological clearly different "portions" of the crystals.

All the measurements were carried out at low temperature $(120 \mathrm{~K})$ on a Bruker APEX II CCD area-detector diffractometer performing short data collection of three sets of exposure frames starting from different $\varphi$ angles, each covering $20^{\circ}$ of rotation in $\Omega$, with a scan step of $0.3^{\circ}$ and an exposure time per frame of $20 \mathrm{~s}$.
Crystals with a size of $1.2-1.6 \mathrm{~mm}$ were mounted with a small amount of oil on a glass fibre and centred in the X-ray beam (beam size of $0.5 \mathrm{~mm}$ ) by using a video camera. A set of different diffraction collection procedures was carried out across different parts of the crystals. Orientation matrices and unit cell parameters were determined for each measurement following the same procedure and applying the same restrictions to the data processing, reflection indexing and unit cell refinement.

Moreover, to assess and compare the degree of order and the crystallinity of the different volumes of the crystals, the half widths of rocking curves of a series of $h k l$ reflections have been analysed.

It has been found that the deformed crystal zones diffract less (about 30\% fewer reflections for the same $I / \sigma$ threshold) and worse (FWHM rocking curves $0.2^{\circ}-0.5^{\circ}$ broader) than the smooth crystal areas, while no significant variations in the unit cell dimensions have been observed.

\section{Results and discussion}

Due to their structural complexity and frequently porous nature, coordination polymers can be difficult to crystallize or they only yield crystals which are too small or not tough enough for complete physical characterization, including the determination of their crystal structure. This is the case for the flexible porous network $\left[\mathrm{Cu}(\mathrm{bpy})_{2}\left(\mathrm{SO}_{3} \mathrm{CF}_{3}\right)_{2}\right]$.solvent (bpy $=4,4^{\prime}$-bipyridine) 1 , with a $\left(4^{2} \cdot 8^{4}\right)$-lvt topology, ${ }^{30}$ crystallized by using two immiscible solvents (water for the copper salt and dichloromethane for the bpy ligand), in the form of small dark blue crystals that dehydrate quickly and lose part of their crystallinity upon removal from the mother liquor. In order to increase the size of the crystals and also to stabilize the samples with respect to solvent loss, we carried out a series of crystallization trials by using TMS polysiloxane gel as the growth medium. Fast screening was performed by changing the gel density, the organic solvent-water ratio in the gelling solutions and the concentrations of the two reactant solutions. Surprisingly, the goal was achieved by using a very low density gel system (5\%) prepared in THF in the presence of the minimum amount of water required for the siloxane hydrolysis reaction. The greatly enhanced stability of these crystals, also at room temperature, has enabled accurate determination of the dynamic behaviour of the system during the desolvation process. The unit cell parameters and the complete crystal structure have been obtained at any point of the solvent release process. To our knowledge, this represents the first example of a very flexible porous coordination network for which it has been possible to characterize the whole phenomenon by using single crystal diffraction. ${ }^{31}$

The unexpected increased resistance of the samples was initially attributed to the incorporation of the silica gel inside the crystals on the basis of the similarity of these porous compounds with "spongy" protein crystal structures that always contain a large amount of water, thus resulting very fragile, difficult to manipulate and often poor diffraction 
quality. As already stated above, many crystals of macromolecules are able to trap large amounts of a gel matrix during the growth process without losing their crystallographic order. The embedded gel framework produces large reinforcement of the crystals, improving their mechanical properties and diffraction quality. This effect was found to be strongly correlated with the gel concentration which, at high values, can also produce morphological deformations of crystals at different scale levels. ${ }^{11,12,32}$

In a similar way, the gel grown crystals of our polymer show an apparently "regular" macroscopic morphology with rough, stepped faces (Fig. 1b) that disguise a peculiar and noteworthy microstructure, recalling the overall array of the crystal structure. This is characterized by intersecting orthogonal pores with large and accessible openings only along the crystallographic $c$ axis (Fig. 1a). Scanning electron microscopy images (Fig. 1c and d) show a grid of irregular square meshes of some microns in size that are clearly the entrances of tunnel cavities. Since this unusual porous microstructure has been observed only for the gel-grown crystals (in TMS-water-THF), we attribute to the gel matrix a templating effect similar to that observed in others systems, ${ }^{4}$ although the mechanism of action is not clear.

Moreover, intra-crystalline modifications, which are responsible for the characteristic porous nanostructures and surface textures observed in inorganic systems as a result of biomineralization processes, are still poorly understood phenomena, but are always attributed to the deposition and entrapment of a macromolecular phase at crystal surfaces. ${ }^{33}$

Unfortunately, in this case, we have not been able either to demonstrate the incorporation of the gel inside the crystals, or to exclude any kind of contamination by the gel polymer. Further studies are in progress to identify the role of the gel
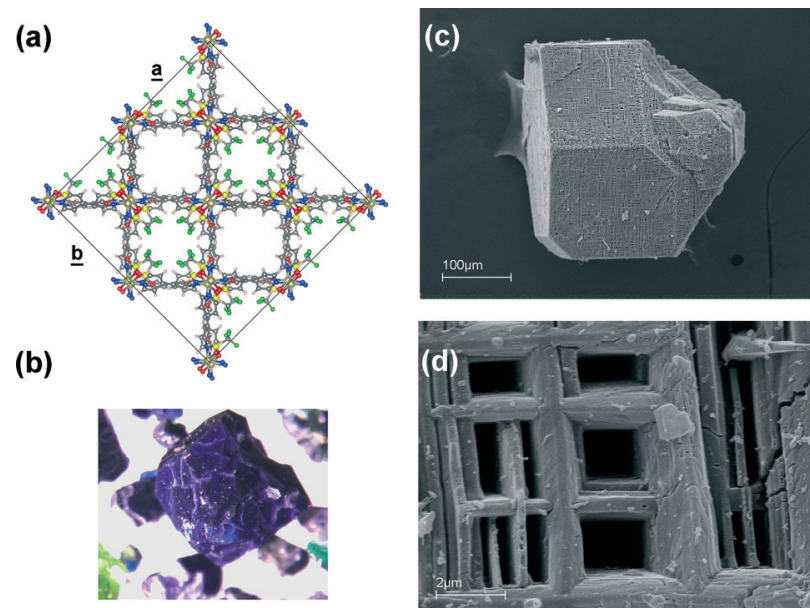

Fig. 1 (a) View along the crystallographic $c$ axis of the 3Dcoordination network $\left[\mathrm{Cu}(\mathrm{bpy})_{2}\left(\mathrm{SO}_{3} \mathrm{CF}_{3}\right)_{2}\right]$. solvent characterized by square nanoporous channels with a chess board distribution. (b) An optical microscopy (OM) image of a crystal of the polymer grown from low density TMS silica-gel; (c, d) scanning electron microscopy (SEM) images of the same crystal showing a microchannel pattern resembling the porous configuration present in the crystal structure.

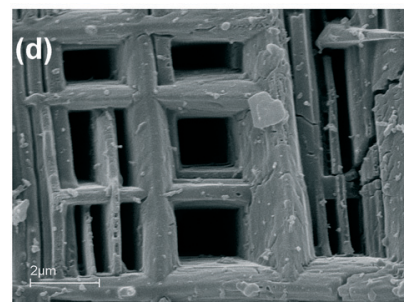

polymer by using other gelling systems which are easier to detect even in trace amounts.

To verify the effectiveness of the improved "elastic behaviour" of the crystalline structure of our gel grown crystals, we performed preliminary single-crystal diffraction measurements at a high-pressure of up to $3.5 \mathrm{GPa}$ using nonpenetrating P-media. ${ }^{34,35}$ Even though only partial data are available, it has been possible to confirm the structural stability of the material in a wide range of pressures and put forward some initial hypothesis on the dynamic behaviour of the network system. In fact, the volumetric compression data show initial oscillation of all the parameters followed by a monotonic decrease of the volume (until about $16 \%$ at $P=3.3$ $\mathrm{GPa}$ ) and of the unit cell axes (of about $2 \%$ for $a$ and $b$, about $12 \%$ for $c$ ) with increasing pressure (Table S1 $\dagger$ ). This unusual and nonlinear trend is probably due to the overall flexibility of the network that, for its peculiar topology, can be contracted or extended in a way that resembles a pantograph, as has been observed during the thermal emptying of the material. This behaviour arises from the tetragonal symmetry (with the space group $I 4_{1} /$ acd) and the orientation of the net with respect to the crystallographic axes, thus allowing the occurrences of shrinking and expansion of the system considering only the variations of the unit cell parameters (Fig. S3†).

Different from the case just described, we found one example in which the inclusion of the gel matrix is evident because the crystals appear totally or partially turbid while maintaining a shapely habit with smooth faces (Fig. 2). The crystals are formed by one-dimensional coordination polymers having the formula $\left[\mathrm{Cd}(\mathrm{bpp})_{3} \mathrm{Cl}_{2}\right] \cdot 2 \mathrm{H}_{2} \mathrm{O}(2),{ }^{23,36}$ which consist of interdigitated zig-zag chains forming a threedimensional array with a high packing density. The inclusion has been observed only in agarose gel prepared at a relatively high concentration (1\%), in the bottom half of the crystallization tube by using the reaction single diffusion technique, with the ligand dissolved in the gel medium and the metal salt in the supernatant solution.

In a previous work, we found that the same metal-ligand system $\left(\mathrm{CdCl}_{2}\right.$-bpp) gave rise to a different polymeric species by using a mixed solvent system (water/methyl or ethyl alcohol) instead of water only. The structure of this polymer, with the general formula $\left[\mathrm{Cd}(\mathrm{bpp})_{2} \mathrm{Cl}_{2}\right] \cdot \operatorname{solv}(3)$, consists of four
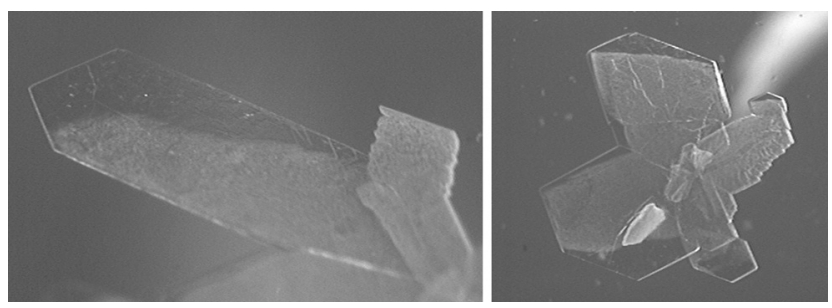

Fig. 2 Crystals of the one dimensional polymer $\left[\mathrm{Cd}(\mathrm{bpp})_{3} \mathrm{Cl}_{2}\right] \cdot 2 \mathrm{H}_{2} \mathrm{O}$ grown from $1 \mathrm{w} / \mathrm{v} \%$ agarose hydrogel; the crystals appear cloudy and opaque due to the inclusion of the gel matrix during growth. Despite of this contamination, the crystals maintain a normally developed habit with smooth faces. 
interpenetrating diamondoid networks (dia) and contains large but isolated cavities filled with solvent molecules. The colourless crystals grown from solution, with a characteristic well-formed bipyramidal habit, are unstable and lose their crystallinity when taken out from the mother liquor. In order to find crystallization conditions which are able to lead to more stable crystals, we have performed restricted crystallization screening in gel by using two- and three-layer diffusion techniques. ${ }^{24,37}$ Although it was only a preliminary experiment, we have observed many interesting phenomena: gelinduced deformation of the crystal morphology, the deep influence of the initial reactant concentrations and, above all, of the gel density on the final equilibrium composition of the system, the quick nucleation and growth at the gel-solution interface of long dendritic plate-like crystals and their morphological evolution.

The two-layer method was carried out in a test tube by gelling a water solution of $\mathrm{CdCl}_{2}$ and then layering on it a methanolic solution of the bpp ligand. Screening was performed by systematically changing the absolute and the relative concentrations of the reactants $(10$ to $30 \mathrm{mM} ; 1: 3$ metal ligand molar ratio) and by using agarose gel prepared at different concentrations $(1 \%, 0.5 \%, 0.1 \%)$. As already observed earlier for analogous Cu-bpp and Co-bpp systems in solution, ${ }^{21}$ this very simple but systematic exploration of the crystallization conditions has revealed the existence of a second crystalline phase characterized by a needle-like morphology which was found to be the already known threedimensional 2-fold interpenetrating network with an sra topology, $\left[\mathrm{Cd}_{2}(\mathrm{bpp})_{3}\left(\mu^{2}-\mathrm{Cl}\right)_{2}(\mu-\mathrm{Cl})_{2}\right]$ (4) reported in ref. 38. This compound was synthesized under hydrothermal conditions only in the presence of ammonium formate, to which they have attributed a significant templating effect. This hypothesis is clearly contradicted by our experimental data.

Optical examination of the crystallization experiments allowed us to construct an approximate "phase" diagram (total concentration $[\mathrm{L}]+[\mathrm{M}]$ vs. $\mathrm{L} / \mathrm{M}$ ratio), ${ }^{39,40}$ that shows predominance of the 3D-diamondoid phase and also a narrow region of coexistence of the two species at low L/M stoichiometric ratios $(<1)$ (Fig. 3). Likewise, analysis of the microcrystalline powder samples prepared from methanol/water solution $(1: 1, \mathrm{v} / \mathrm{v})$ at different concentrations of the reactants (see the experimental section) has confirmed the formation of an sra-dia multiphase mixture at low ligand-metal ratios $(<1: 1)$, showing the possibility of obtaining a second species, at least in a massive form, also from solution.

A very important aspect is represented by the observation that a decrease in the gel concentration seems to cause enlargement of the two-phase field of existence, until, at a very low gel density, a potential area of existence of the second phase alone is found. An analogous effect due to the gel density has been observed in a similar study on copolymerization of lanthanide ions with polycarboxylate anions as ligands. The phase diagram constructed as a function of the reactant concentration and the gel density clearly shows dependence on both these parameters. ${ }^{41}$

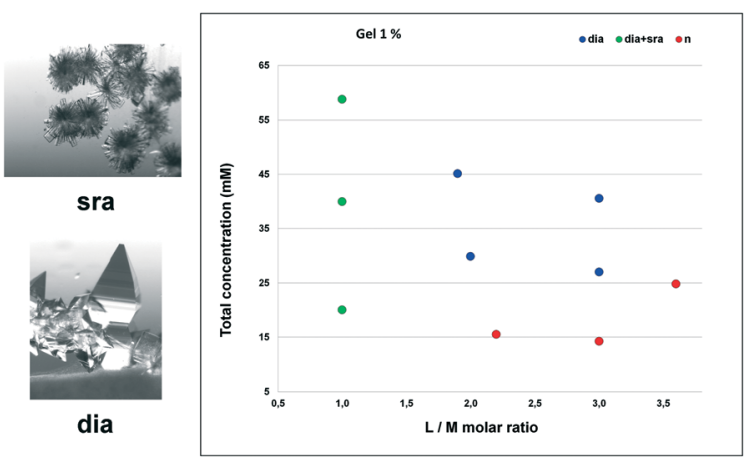

Fig. 3 Approximate equilibrium phase diagram for the $\mathrm{CdCl}_{2} / \mathrm{bpp}$ system constructed from the results of crystallization screening performed by using the two layer-gel technique (agar gel-MeOH). Each array item has been repeated three times. The stability regions of the different species as a function of $[\mathrm{L}]+[\mathrm{M}]$ total initial concentration and $[\mathrm{L}] /[\mathrm{M}]$ ratio $\left(\mathrm{M}=\mathrm{Cd}^{2+} ; \mathrm{L}=\mathrm{bpp}\right)$ are shown in colors (3Ddiamontoid topology $($ dia $)=$ blue dia + sra $=$ green, no crystals $=$ red $)$.

This systematic analysis should be obviously extended and compared with the results of deep crystallization screening in solution in order to understand the nature of the relative stability of the two phases and fully clarify the effect of the gel on the system.

It is important to highlight for coordination polymers, as well as for more simple inorganic systems, how the choice of the $[\mathrm{L}]+[\mathrm{M}]$ and $\mathrm{L} / \mathrm{M}$ parameters for the construction of the phase diagram has been fundamental to acquire deep knowledge on the system's behaviour involving different competing crystalline phases, as demonstrated in other previous cases. $^{24,41,42}$ The use of gel as diffusion media, which usually requires a higher degree of supersaturation for overcoming the energy barrier of both nucleation and growth, can deeply affect the phase diagram, promoting the nucleation of metastable phases or generating a variety of crystal morphologies. ${ }^{43,44}$

A more comprehensive and detailed study is in progress in order to investigate the effects on a system generated by modifications of the crystallization configuration (like insertion of an intermediate chamber of inert gel between two reactant solutions or metal-ligand inversion in layering) and of growth media composition (gel nature and gel density).

In addition to the above-discussed findings, thanks to the screening experiment, we have found that in most crystallization trials, crystals of one or both species ( 3 and 4 ) were present in the supernatant solution due to the reverse diffusion of the reactants. Moreover, single crystals as well as a cluster of crystals, were often found at the gel-solution interface where the concentration gradients are large. In particular, crystals of compound 3 with a characteristic bipyramidal shape were grown at the gel/solution interface, maintaining their single crystal nature; however, the bottom part of the crystals grown in the gel appeared clearly deformed, with a hugely elongated shape and a rugged surface (Fig. 4). It is reasonable to attribute these macro- and micro-morphological modifications to the pressure exerted by the gel that, nevertheless, does not 
seem to be trapped in the crystal during the growth process, despite the low lattice density, the porosity of the crystal structure and the very fast growth rate of the crystals. However, we did not observe a "stabilizing effect" of the gel on the crystals that, similar to those grown from solution, lost quickly the solvent molecules becoming opaque and brittle.

The main role of the gel in the morphological deformation of the crystals, that could also be attributed to one of the components present in the stoichiometric excess, has been confirmed by the disappearance of any deformation at a very low gel concentration $(0.1 \% \mathrm{w} / \mathrm{v})$, while maintaining all the other crystallization parameters constant.

For selection of the samples, rocking curve measurements were carried out on different parts of the crystal volume, confirming clear but limited degradation of the crystal quality due to the gel effect. Indeed, the diffraction peaks of the gel grown parts of the crystals were clearly broader compared to those relative to portions of the same crystals grown from solution. Nevertheless, in spite of the crystal deformation, no changes have been observed in the unit cell values.

In general, on the basis of what has also been experimentally observed with other coordination compounds, we can state that an increase in gel density induces evolution of crystals toward morphological instability from specific polyhedral habits to dendrites, regardless of the type of crystallizing materials and gelling agent used. ${ }^{15,45}$

Lastly, the gel technique has been proven helpful to perform nucleation/growth kinetic studies. Kinetic investigation was firstly carried out on the $1 \mathrm{D}$ polymeric $\mathrm{CdCl}_{2}$-bpp system 2 stated above. The product was crystallized by layering a water solution of the metal salt on a gelled aqueous solution of the ligand in a test tube under different conditions (gel density, M/L molar ratio and total concentration). We observed that in all the crystallization trials, nucleation takes place at the gel-sol interface and then the crystals grow quickly and continuously in the gel up to several centimetres in length. The final size and the morphology of the crystals have been shown to be largely dependent on the crystallization parameters, especially on the gel density; however, a

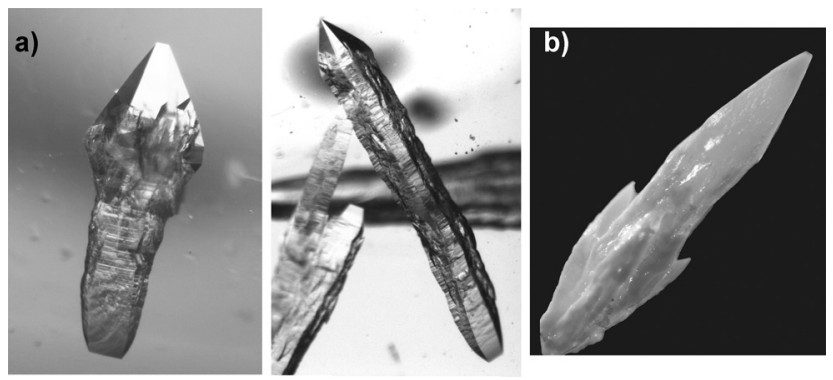

Fig. 4 (a) Crystals of the porous diamontoid 3D network $\left[\mathrm{Cu}(\mathrm{bpp})_{2} \mathrm{Cl}_{2}\right]$ $\cdot \mathrm{H}_{2} \mathrm{O}$ grown at the solution-gel interface. The upper part of the crystals, grown in solution, maintains its pyramidal habit unaltered, while the bottom part, grown in gel, appeared clearly deformed with an elongated shape. (b) If removed from the growth solution and dried in the air, the crystals lost solvent molecules and became clearly amorphous. more systematic exploration of all the experimental factors, including the effect of the gelling agent, has yet to be performed.

Thanks to the straightforward configuration of the growing system, we followed the growth process by in situ optical microscopy in order to determine the growth rate as a function of time and thus obtain information on the kinetic behaviour of the system and on the surface diffusion processes involved. Under the conditions used for the kinetic experiment, the crystals grew with a characteristic dendritic fernleaf shape, which is formed by overlapping crystals that express a geometry consistent with the (001) plate form of faceted crystals.

Data have been collected from two different crystals. The results are illustrated in Fig. 5 and S5.† In all the graphs, only the data for the middle and last stage of the growth process are reported. The initial portion of the second curve in Fig. 5 ( $L$ vs. $t$ ) is parabolic, as confirmed by the linear dependence of $L^{2}$ from the time of growth (see last graph in Fig. S5 $\dagger$ ). This behaviour is considered to be characteristic of a one dimensional diffusion controlled process. ${ }^{46,47}$ Moreover, at the very late stage of growth, a departure from linearity is observed and the curve is better described by a quadratic function that seems to suggest a growth mechanism controlled by surface integration.

In any case, it is clear that relaxation of the supersaturated system toward equilibrium causes a morphological change in the system. The images in Fig. 6 show the morphological evolution, from a dendritic shape to a more regular form, undergone by the crystals during the growth process that reveals "reverse" kinetic roughening transition and therefore a change in the growth mechanism. This phenomenon can be explained by considering the morphological evolution of the surface from smooth to rough with the increase in supersaturation and the correlation of the driving force with the growth rate. ${ }^{47,48}$

The initial rough macro-surface of the crystal starts to become more regular as the growth slows down, along with the formation of defined macrosteps. As the growth proceeds,

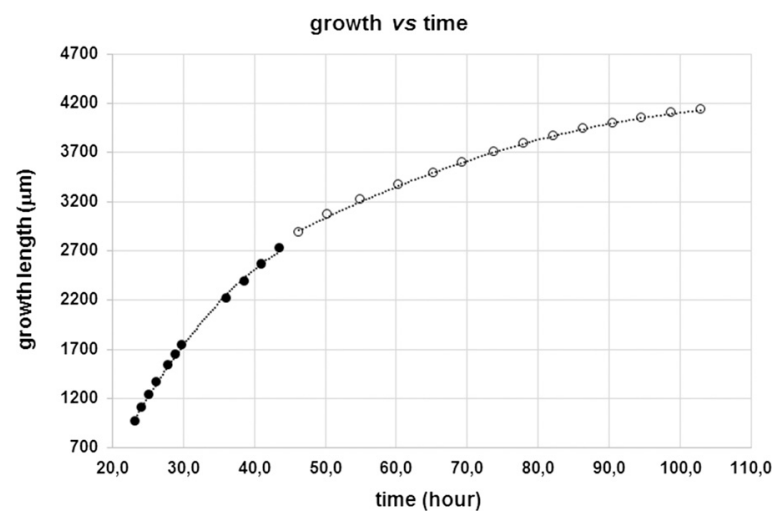

Fig. 5 Crystal length variations as a function of growth time. The shape of the curve changes over time (black and white dots) suggesting a change in the growth mechanism. 


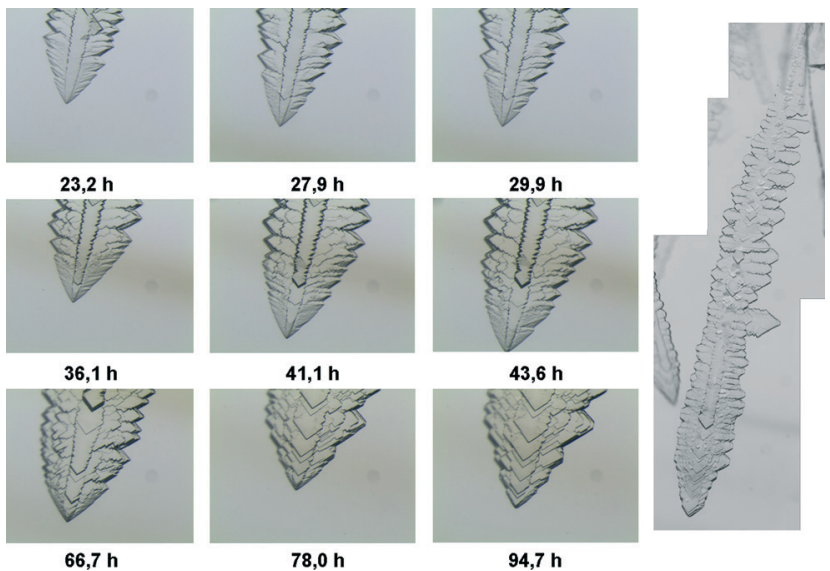

Fig. 6 Optical microscopy images of the morphology evolution in the time-dependent crystal growth experiment of the coordination network 2 (left). Final morphology of the entire dendritic crystal (right).

lateral propagation of the steps takes place until, below a threshold value of supersaturation, a layer-by-layer type mechanism takes over, leading to morphological restoration of the crystals. ${ }^{46,49}$

To confirm the suitability of the proposed kinetic model, the correlation between the mass gradient and the reactant concentration towards crystal growth should be established, along with accurate characterization of the micromorphology of the surface of the crystal faces.

Actually, we have already reported the results of a crystal growth study at the microscale, performed by in situ atomic force microscopy, on the isomorphous copper compound of the $1 \mathrm{D}$ polymeric species $2 .^{50}$ However, the investigation was carried out at low supersaturation and, as expected, spiral growth was the only growth mechanism observed, while no evidence for 2D nucleation was found.

\section{Conclusions}

In summary, we have presented some preliminary but sound results obtained by applying the gel technique to the crystallization of coordination polymers, which are a very special class of materials characterized by huge structural diversity and wide chemical versatility. Beyond the possibilities to induce new phases, as already demonstrated in the past, this work shows how the gel growth method could represent a powerful tool to study the crystallogenesis of polymeric species, including kinetic and thermodynamic aspects operative in phase selection, as well as an alternative route to induce morphological and structural modifications.

In addition, we have described some noteworthy effects on the physical properties and the functional performance of the gel grown crystals of coordination networks, opening up new possibilities for their technological applications. Indeed, the most important finding of our investigations is represented by the characteristic porous nanostructure recalling the void channel distribution of the net topology observed in some gel-grown crystals. This microstructure, besides being responsible for changes in material properties, show important similarities to the peculiar surface textures observed in inorganic systems involved in biomineralization processes.

\section{Acknowledgements}

We gratefully acknowledge financial support from Fondazione Cariplo (grant no: 2012-0921).

\section{References}

1 S. Mann, Angew. Chem., Int. Ed., 2000, 39, 3392-3406.

2 B. Pokroy, A. N. Fitch, F. Marin, M. Kapon, N. Adir and E. Zolotoyabko, J. Struct. Biol., 2006, 155, 96-103.

3 J. S. Robach, S. R. Stock and A. Veis, J. Struct. Biol., 2005, 151, 18-29.

4 H. Li and L. A. Estroff, CrystEngComm, 2007, 9, 1153.

5 J. A. Gavira and J. M. Garcia-Ruiz, Acta Crystallogr., Sect. D: Biol. Crystallogr., 2002, 58, 1653-1656.

6 L. A. Estroff, H. Li, M. Kunitake, H. L. Xin, A. Vodnick, S. P. Baker and D. A. Muller, in Abstracts of Papers, 237th ACS National Meeting, March 22-26, 2009, American Chemical Society, Salt Lake City, UT, United States, 2009, p. CHED1153.

7 O. Grassmann, R. B. Neder, A. Putnis and P. Lobmann, Am. Mineral., 2003, 88, 647-652.

8 A. N. Kulak, P. Iddon, Y. Li, S. P. Armes, H. Colfen, O. Paris, R. M. Wilson and F. C. Meldrum, J. Am. Chem. Soc., 2007, 129, 3729-3736.

9 H. Li, H. L. Xin, D. A. Muller and L. A. Estroff, Science, 2009, 326, 1244-1247.

$10 \mathrm{H}$. Li and L. A. Estroff, Adv. Mater., 2009, 21, 470-473.

11 J. M. Garcia-Ruiz, J. A. Gavira, F. Otalora, A. Guasch and M. Coll, Mater. Res. Bull., 1998, 33, 1593-1598.

12 C. Biertuempfel, J. Basquin, D. Suck and C. Sauter, Acta Crystallogr., Sect. D: Biol. Crystallogr., 2002, 58, 1657-1659.

13 L. A. Estroff and A. D. Hamilton, Chem. Mater., 2001, 13, 3227-3235.

14 R. I. Petrova and J. A. Swift, Cryst. Growth Des., 2002, 2, 573-578.

15 Y. Oaki and H. Imai, Cryst. Growth Des., 2003, 3, 711-716.

16 Y. Diao, K. E. Whaley, M. E. Helgeson, M. A. Woldeyes, P. S. Doyle, A. S. Myerson, T. A. Hatton and B. L. Trout, J. Am. Chem. Soc., 2012, 134, 673-684.

17 J. Mahieux, M. Sanselme and G. Coquerel, Cryst. Growth Des., 2013, 13, 908-917.

18 C. Dey, T. Kundu, B. P. Biswal, A. Mallick and R. Banerjee, Acta Crystallogr., Sect. B: Struct. Sci., Cryst. Eng. Mater., 2014, 70, 3-10.

19 N. Stock and S. Biswas, Chem. Rev., 2012, 112, 933-969.

20 Y. Cai, A. R. Kulkarni, Y.-G. Huang, D. S. Sholl and K. S. Walton, Cryst. Growth Des., 2014, 14, 6122-6128.

21 D. Zhao, D. J. Timmons, D. Yuan and H.-C. Zhou, Acc. Chem. Res., 2011, 44, 123-133.

22 S. L. James, Chem. Soc. Rev., 2003, 32, 276-288. 
23 L. Carlucci, G. Ciani, J. M. Garcia-Ruiz, M. Moret, D. M. Proserpio and S. Rizzato, Cryst. Growth Des., 2009, 9, 5024-5034.

24 J. M. Garcia-Ruiz, Key Eng. Mater., 1991, 58, 87-106.

25 http://www.trianatech.com.

26 H. K. Mao, J. Xu and P. M. Bell, J. Geophys. Res.: Space Phys., 1986, 91, 4673.

27 J. C. Chervin, B. Canny and M. Mancinelli, High Pressure Res., 2001, 21, 305-314.

28 M. Merlini and M. Hanfland, High Pressure Res., 2013, 33, 511-522.

29 Agilent Xcalibur CCD System, Crysalis Software. System, 2012.

30 L. Carlucci, N. Cozzi, G. Ciani, M. Moret, D. M. Proserpio and S. Rizzato, Chem. Commun., 2002, 1354-1355.

31 Rizzato, et al., Unpublished raw data.

32 F. Otalora, J. A. Gavira, B. Capelle and J. M. Garcia-Ruiz, Acta Crystallogr., Sect. D: Biol. Crystallogr., 1999, 55, 650-655.

33 E. P. Chang, G. Williamson and J. S. Evans, Cryst. Growth Des., 2015, 15, 1577-1582.

34 E. C. Spencer, R. J. Angel, N. L. Ross, B. E. Hanson and J. A. K. Howard, J. Am. Chem. Soc., 2009, 131, 4022-4026.

35 S. R. Madsen, J. Overgaard, D. Stalke and B. B. Iversen, Dalton Trans., 2015, 44, 9038-9043.

36 L. Carlucci, G. Ciani, M. Moret, D. M. Proserpio and S. Rizzato, Chem. Mater., 2002, 14, 12-16.

37 J. M. Garcia-Ruiz, Methods Enzymol., 2003, 368, 130-154.

38 F. Luo, Y. Che and J. Zheng, Inorg. Chem. Commun., 2006, 9, 856-858.
39 S. D. Durbin and G. Feher, Annu. Rev. Phys. Chem., 1996, 47, 171-204.

40 N. E. Chayen, T. J. Boggon, A. Cassetta, A. Deacon, T. Gleichmann, J. Habash, S. J. Harrop, J. R. Helliwell, Y. P. Nieh, M. R. Peterson, J. Raftery, E. H. Snell, A. Hadener, A. C. Niemann, D. P. Siddons, V. Stojanoff, A. W. Thompson, T. Ursby and M. Wolff, Q. Rev. Biophys., 1996, 29, 227-278 4 plates.

41 C. Daiguebonne, O. Guillou and K. Boubekeur, Inorg. Chim. Acta, 2000, 304, 161-169.

42 C. Daiguebonne, A. Deluzet, M. Camara, K. Boubekeur, N. Audebrand, Y. Gerault, C. Baux and O. Guillou, Cryst. Growth Des., 2003, 3, 1015-1020.

43 M. Pauchet, T. Morelli, S. Coste, J.-J. Malandain and G. Coquerel, Cryst. Growth Des., 2006, 6, 1881-1889.

44 M.-C. Robert, O. Vidal, J.-M. Garcia-Ruiz and F. Otalora, in Cryst. Nucleic Acids. Proteins, 2nd edn, Oxford University Press, 1999, pp. 149-175.

45 P. Gao, M. Gu and L.-L. Xiao, Cryst. Res. Technol., 2008, 43, 496-501.

46 C. C. Desai and J. L. Rai, Surf. Technol., 1982, 15, 25-32.

47 A. M. E. Raj, D. D. Jayanthi, V. B. Jothy, M. Jayachandran and C. Sanjeeviraja, Cryst. Res. Technol., 2008, 43, 1307-1313.

48 J. M. Garcia-Ruiz and A. Moreno, J. Cryst. Growth, 1997, 178, 393-401.

49 P. Cubillas and M. W. Anderson, in Zeolites and Catalysis, Wiley-VCH Verlag GmbH \& Co. KGaA, 2010, pp. 1-55.

50 M. Moret and S. Rizzato, Cryst. Growth Des., 2009, 9, 5035-5042. 